TRANSACTIONS OF THE

AMERICAN MATHEMATICAL SOCIETY

Volume 349, Number 5, May 1997, Pages 1769-1782

S 0002-9947(97)01781-9

\title{
REVERSION OF POWER SERIES AND THE EXTENDED RANEY COEFFICIENTS
}

\author{
CHARLES CHING-AN CHENG, JAMES H. McKAY, JACOB TOWBER, \\ STUART SUI-SHENG WANG, AND DAVID L. WRIGHT
}

\begin{abstract}
In direct as well as diagonal reversion of a system of power series, the reversion coefficients may be expressed as polynomials in the coefficients of the original power series. These polynomials have coefficients which are natural numbers (Raney coefficients). We provide a combinatorial interpretation for Raney coefficients. Specifically, each such coefficient counts a certain collection of ordered colored trees. We also provide a simple determinantal formula for Raney coefficients which involves multinomial coefficients.
\end{abstract}

Let $F_{1}, \ldots, F_{n}$ be polynomials in variables $x_{1}, \ldots, x_{n}$ with complex coefficients, where $n \geqslant 2$. Suppose, for each $i, F_{i}=x_{i}+$ higher degree terms and the Jacobian determinant of $F_{1}, \ldots, F_{n}$ is equal to 1 . Then the Jacobian Conjecture [1], [9] asserts, in this case, that $x_{1}, \ldots, x_{n}$ are also polynomials in $F_{1}, \ldots, F_{n}$ with complex coefficients. This long-standing conjecture has not been solved even for $n=2$. Since it can be proved that $x_{1}, \ldots, x_{n}$ are (formal) power series in $F_{1}, \ldots, F_{n}$ with complex coefficients, the Jacobian Conjecture asserts that these power series are really polynomials. This provides the motivation for this paper.

Let $F_{1}, \ldots, F_{n}$ be power series in variables $x_{1}, \ldots, x_{n}$ of the form $F_{i}=x_{i}+$ higher degree terms with indeterminate coefficients for each $i$. It is known (e.g. [2, Chapter III, Section 4.4, Proposition 5, p. 219]) that $F=\left(F_{1}, \ldots, F_{n}\right)$ has a (unique) compositional inverse, i.e., there exists $G=\left(G_{1}, \ldots, G_{n}\right)$ where each $G_{i}$ is a power series in variables $x_{1}, \ldots, x_{n}$ such that $F \circ G=1$ and $G \circ F=1$, or equivalently, $F_{i}\left(G_{1}, \ldots, G_{n}\right)=x_{i}$ and $G_{i}\left(F_{1}, \ldots, F_{n}\right)=x_{i}$ for all $i$. There are various methods in the literature to find the coefficients of $G_{i}$. In this paper we shall present two new ones. Since each coefficient of $G_{i}$ is a polynomial in the indeterminate coefficients of $F_{1}, \ldots, F_{n}$, it is enough to find the coefficients of these polynomials. We will refer to these coefficients as the (extended) Raney coefficients. In the first method, generating functions in infinitely many variables are used to show that each Raney coefficient has a combinatorial interpretation as the number of colored trees in a certain collection (Theorems 2.4 and 2.5). In

Received by the editors April 4, 1994.

1991 Mathematics Subject Classification. Primary 13F25, 05A15, 05C05, 13P99; Secondary $32 \mathrm{~A} 05$.

Key words and phrases. Reversion of power series, direct reversion, diagonal reversion, Jacobian conjecture, colored trees, colored forests, inventory, Raney coefficients, Laurent series, Jacobi's residue formula.

The third author was supported in part by the National Science Foundation under Grant DMS-9012210.

The fifth author was supported in part by the National Security Agency under Grant MDA904-89-H-2049. 
the second method, Jacobi's residue formula, in a more general setting (Theorem 1.4), is used to obtain a determinantal formula for each Raney coefficient (Corollary 2.7). Consequently this gives a formula for counting certain colored forests. These generalize Raney's results in [7] where $n=1$.

\section{Formal Laurent SERIES AND Jacobi's Residue Formula}

Let $R$ be a commutative ring with identity and let $\mathbb{Z}$ denote the ring of integers. A Laurent monomial in variables $x_{1}, \ldots, x_{n}$ is a power product $x_{1}^{j_{1}} x_{2}^{j_{2}} \cdots x_{n}^{j_{n}}$ where each exponent $j_{i}$ is in $\mathbb{Z}$. The degree of this monomial is the sum of its exponents. A homogeneous Laurent polynomial over $R$ in variables $x_{1}, \ldots, x_{n}$ is an $R$-linear combination of (finitely many) Laurent monomials of the same degree. A formal Laurent series in variables $x_{1}, \ldots, x_{n}$ with coefficients in $R$ is an expression of the form

$$
F=\sum_{k=-\infty}^{\infty} f_{k}
$$

where each $f_{k}$ is either 0 or a homogeneous Laurent polynomial of degree $k$ in $x_{1}, \ldots, x_{n}$ with coefficients in $R$, and there exists an integer $p$ such that $f_{k}=0$ for all $k<p$. In this case we shall call $f_{k}$ the homogeneous component of $F$ of degree $k$. The order of a nonzero formal Laurent series $F$ is defined to be the smallest integer $p$ such that $f_{p} \neq 0$. In this case, we shall call $f_{p}$ the initial part of $F$. The set $R\left(\left(\left(x_{1}, \ldots, x_{n}\right)\right)\right)^{1}$ of all formal Laurent series forms a commutative ring with identity under the obvious addition and multiplication. Note that if $F$ and $G$ have orders $p$ and $q$ respectively, then their product has order at least $p+q$. If, in addition, $R$ is a field, then $F G$ has order precisely $p+q$.

Remark. Let $R\left[\left[x_{1}, \ldots, x_{n}\right]\right]_{x_{1} x_{2} \cdots x_{n}}$ denote the localization of the power series ring $R\left[\left[x_{1}, \ldots, x_{n}\right]\right]$ at the multiplicative set $\left\{1,\left(x_{1} x_{2} \cdots x_{n}\right),\left(x_{1} x_{2} \cdots x_{n}\right)^{2}, \ldots\right\}$. Note that if $n \geqslant 2$, then

$$
R\left[\left[x_{1}, \ldots, x_{n}\right]\right]_{x_{1} x_{2} \ldots x_{n}} \varsubsetneqq R\left(\left(\left(x_{1}, \ldots, x_{n}\right)\right)\right),
$$

as $\sum_{k=0}^{\infty} x_{1}^{2 k+1} x_{2}^{-k}$ is not in the former but in the latter; although

$$
R\left[\left[x_{1}\right]\right]_{x_{1}}=R\left(\left(\left(x_{1}\right)\right)\right) .
$$

Lemma 1.1. Let $F \in R\left(\left(\left(x_{1}, \ldots, x_{n}\right)\right)\right)$ and let $f_{p}$ be the initial part of $F$. Consider the following conditions.

1. F has a multiplicative inverse in $R\left(\left(\left(x_{1}, \ldots, x_{n}\right)\right)\right)$.

2. $f_{p}$ has a multiplicative inverse in $R\left(\left(\left(x_{1}, \ldots, x_{n}\right)\right)\right)$.

3. $f_{p}$ consists of a single term with invertible coefficient.

Then $3 \Rightarrow 2 \Rightarrow 1$.

Proof. $3 \Rightarrow 2$. If $f_{p}=a x_{1}^{j_{1}} x_{2}^{j_{2}} \cdots x_{n}^{j_{n}}$ with $a$ invertible in $R$, then $f_{p}$ has multiplicative inverse $a^{-1} x_{1}^{-j_{1}} x_{2}^{-j_{2}} \cdots x_{n}^{-j_{n}}$ in $R\left(\left(\left(x_{1}, \ldots, x_{n}\right)\right)\right)$.

$2 \Rightarrow 1$. If $f_{p}$ is invertible in $R\left(\left(\left(x_{1}, \ldots, x_{n}\right)\right)\right)$, then

$$
F=\sum_{k=p}^{\infty} f_{k}=f_{p}\left[1+f_{p}^{-1} \sum_{k=p+1}^{\infty} f_{k}\right]=f_{p}[1-H]
$$

\footnotetext{
${ }^{1}$ Thanks are due to Boo Barkee for this eye-catching notation.
} 
where $H=(-1) f_{p}^{-1} \sum_{k=p+1}^{\infty} f_{k}$ is a formal Laurent series. Since $\operatorname{order}\left(f_{p}^{-1}\right)=-p$, it follows that $\operatorname{order}(H) \geqslant 1$. Hence $\operatorname{order}\left(H^{k}\right) \geqslant k$ and therefore $1+H+H^{2}+\cdots \in$ $R\left(\left(\left(x_{1}, \ldots, x_{n}\right)\right)\right)$. Thus $F$ is invertible with inverse $f_{p}^{-1}\left(1+H+H^{2}+\cdots\right)$.

Remark. In case $R$ is a field the three conditions above are equivalent.

The residue of a formal Laurent series $F$ in $R\left(\left(\left(x_{1}, \ldots, x_{n}\right)\right)\right)$ is the coefficient of $x_{1}^{-1} x_{2}^{-1} \cdots x_{n}^{-1}$ in $F$. Note that residue is additive in the sense that if an infinite sum $\sum_{i \in I} G_{i}$ makes sense, then

$$
\text { residue }\left(\sum_{i \in I} G_{i}\right)=\sum_{i \in I} \operatorname{residue}\left(G_{i}\right)
$$

In what follows we let

$$
\frac{\partial\left(H_{1}, \ldots, H_{n}\right)}{\partial\left(x_{1}, \ldots, x_{n}\right)}
$$

be the Jacobian determinant of $H_{1}, \ldots, H_{n} \in R\left(\left(\left(x_{1}, \ldots, x_{n}\right)\right)\right)$ with respect to $x_{1}, \ldots, x_{n}$.

Lemma $1.2([9$, pp. $471-472])$. Let $\llbracket H_{1}, \ldots, H_{n} \rrbracket=\frac{\partial\left(H_{1}, \ldots, H_{n}\right)}{\partial\left(x_{1}, \ldots, x_{n}\right)}$. Then

1. $\llbracket, \ldots, \rrbracket$ is R-multilinear.

2. 【 $, \ldots, \rrbracket$ is alternating, i.e., $\llbracket H_{1}, \ldots, H_{n} \rrbracket=0$ if there exist $i \neq j$ such that $H_{i}=H_{j}$.

3. 【, ,., \is anticommutative, i.e.,

$$
\llbracket H_{1}, \ldots, H_{i}, \ldots, H_{j}, \ldots, H_{n} \rrbracket=-\llbracket H_{1}, \ldots, H_{j}, \ldots, H_{i}, \ldots, H_{n} \rrbracket .
$$

4. (Product Rule) $\llbracket F_{1} G_{1}, H_{2}, \ldots, H_{n} \rrbracket=F_{1} \llbracket G_{1}, H_{2}, \ldots, H_{n} \rrbracket+G_{1} \llbracket F_{1}, H_{2}, \ldots, H_{n} \rrbracket$.

5. (Power Rule) For any integer $m$,

$$
\llbracket H_{1}^{m}, H_{2}, \ldots, H_{n} \rrbracket=m H_{1}^{m-1} \llbracket H_{1}, H_{2}, \ldots, H_{n} \rrbracket .
$$

(Here we require that $H_{1}$ be invertible if $m$ is negative.)

6. If $H$ is invertible, then $\llbracket H^{-1}, H, H_{3}, \ldots, H_{n} \rrbracket=0$.

Lemma 1.3. residue $\left(\frac{\partial\left(F_{1}, \ldots, F_{n}\right)}{\partial\left(x_{1}, \ldots, x_{n}\right)}\right)=0$.

Proof. By 1 of Lemma 1.2 and (1.2), it is enough to examine the case where each $F_{i}$ consists of a single term:

$$
F_{i}=a_{i} x_{1}^{b_{i 1}} x_{2}^{b_{i 2}} \cdots x_{n}^{b_{i n}} .
$$

Factoring $a_{i} x_{1}^{b_{i 1}} x_{2}^{b_{i 2}} \cdots x_{n}^{b_{i n}}$ from the $i^{\text {th }}$ row of the Jacobian matrix for all $i$, and then factoring $x_{j}^{-1}$ from the $j^{\text {th }}$ column for all $j$, we have

$$
\frac{\partial\left(F_{1}, \ldots, F_{n}\right)}{\partial\left(x_{1}, \ldots, x_{n}\right)}=a_{1} \cdots a_{n}\left|\begin{array}{cccc}
b_{11} & b_{12} & \ldots & b_{1 n} \\
b_{21} & b_{22} & \ldots & b_{2 n} \\
\vdots & \vdots & & \vdots \\
b_{n 1} & b_{n 2} & \ldots & b_{n n}
\end{array}\right| x_{1}^{-1+\sum b_{i 1}} \cdots x_{n}^{-1+\sum b_{i n}}
$$

Suppose $\sum b_{i 1}=\cdots=\sum b_{i n}=0$. Then the displayed determinant is 0 and therefore the residue is 0 . Otherwise, at least one of the $x_{i}$ 's has exponent $\neq-1$ and so the residue is 0 by definition. 
Theorem 1.4 (Jacobi's Residue Formula). Suppose, for each $i=1, \ldots, n, F_{i} \in$ $R\left(\left(\left(x_{1}, \ldots, x_{n}\right)\right)\right)$ is of the form

$$
F_{i}=a_{i} x_{1}^{b_{i 1}} x_{2}^{b_{i 2}} \cdots x_{n}^{b_{i n}}+\text { higher degree terms }
$$

with $a_{i}$ invertible in $R$. Then

$$
\begin{aligned}
& \text { residue }\left(F_{1}^{e_{1}} F_{2}^{e_{2}} \ldots F_{n}^{e_{n}} \frac{\partial\left(F_{1}, \ldots, F_{n}\right)}{\partial\left(x_{1}, \ldots, x_{n}\right)}\right) \\
& =\left\{\begin{array}{llc}
\left|\begin{array}{ccc}
b_{11} & \ldots & b_{1 n} \\
\vdots & & \vdots \\
b_{n 1} & \ldots & b_{n n}
\end{array}\right|, \quad \text { if } e_{1}=e_{2}=\cdots=e_{n}=-1, \\
0, & & \text { otherwise. }
\end{array}\right.
\end{aligned}
$$

Proof. We first consider the case $e_{1}=e_{2}=\cdots=e_{n}=-1$. Following the proof of Lemma 1.3, we have

$$
\begin{aligned}
\frac{\partial\left(F_{1}, \ldots, F_{n}\right)}{\partial\left(x_{1}, \ldots, x_{n}\right)}= & a_{1} \cdots a_{n}\left|\begin{array}{ccc}
b_{11} & \ldots & b_{1 n} \\
\vdots & & \vdots \\
b_{n 1} & \ldots & b_{n n}
\end{array}\right| x_{1}^{-1+\sum b_{i 1}} \cdots x_{n}^{-1+\sum b_{i n}} \\
& + \text { higher degree terms. }
\end{aligned}
$$

Since $a_{i}$ is invertible, by the proof of Lemma 1.1, we have

$$
F_{i}^{-1}=a_{i}^{-1} x_{1}^{-b_{i 1}} x_{2}^{-b_{i 2}} \cdots x_{n}^{-b_{i n}}+\text { higher degree terms }
$$

for each $i$. Hence the result follows.

Consider now the remaining case. Permuting the $F_{i}$ and using 3 of Lemma 1.2, we may assume that $e_{1} \neq-1, \ldots, e_{j} \neq-1$, but $e_{j+1}=\cdots=e_{n}=-1$, for some $j$, $1 \leqslant j \leqslant n$. Setting $G_{i}=\frac{1}{e_{i}+1} F_{i}^{e_{i}+1}$ and using 5,1 and, successively, 4, 6 and 3 of Lemma 1.2, we have

$$
\begin{aligned}
& F_{1}^{e_{1}} F_{2}^{e_{2}} \cdots F_{n}^{e_{n}} \llbracket F_{1}, \ldots, F_{n} \rrbracket \\
&=\frac{1}{e_{1}+1} \cdots \frac{1}{e_{j}+1} F_{j+1}^{-1} \cdots F_{n}^{-1} \llbracket F_{1}^{e_{1}+1}, \ldots, F_{j}^{e_{j}+1}, F_{j+1}, \ldots, F_{n} \rrbracket \\
&=F_{j+1}^{-1} \cdots F_{n}^{-1} \llbracket G_{1}, \ldots, G_{j}, F_{j+1}, \ldots, F_{n} \rrbracket \\
&=F_{j+2}^{-1} \cdots F_{n}^{-1} \llbracket F_{j+1}^{-1} G_{1}, \ldots, G_{j}, F_{j+1}, \ldots, F_{n} \rrbracket \\
&=F_{j+3}^{-1} \cdots F_{n}^{-1} \llbracket F_{j+1}^{-1} F_{j+2}^{-1} G_{1}, \ldots, G_{j}, F_{j+1}, \ldots, F_{n} \rrbracket \\
& \cdots \ldots \ldots, \ldots, F_{n} \rrbracket . \\
&= \llbracket F_{j+1}^{-1} F_{j+2}^{-1} F_{j+3}^{-1} \ldots F_{n}^{-1} G_{1}, \ldots, F_{j+1}, \ldots, F_{j}
\end{aligned}
$$

The result now follows from Lemma 1.3.

Corollary 1.5. Suppose, for each $i=1, \ldots, n, F_{i} \in R\left(\left(\left(x_{1}, \ldots, x_{n}\right)\right)\right)$ is of the form

$$
F_{i}=a_{i} x_{i}+\text { higher degree terms }
$$

with $a_{i}$ invertible in $R$. Suppose also that for nonnegative integers $p_{1}, \ldots, p_{n}$,

$$
x_{1}^{p_{1}} x_{2}^{p_{2}} \cdots x_{n}^{p_{n}}=\sum_{l_{1}, \ldots, l_{n}} d_{l_{1}, \ldots, l_{n}}^{p_{1}, \ldots, p_{n}} F_{1}^{l_{1}} F_{2}^{l_{2}} \cdots F_{n}^{l_{n}},
$$


where $d_{l_{1}, \ldots, l_{n}}^{p_{1}, \ldots, p_{n}} \in R$. Then

$$
d_{m_{1}, \ldots, m_{n}}^{p_{1}, \ldots, p_{n}}=\text { residue }\left(x_{1}^{p_{1}} x_{2}^{p_{2}} \cdots x_{n}^{p_{n}} F_{1}^{-m_{1}-1} \cdots F_{n}^{-m_{n}-1} \frac{\partial\left(F_{1}, \ldots, F_{n}\right)}{\partial\left(x_{1}, \ldots, x_{n}\right)}\right) .
$$

Proof.

$$
\begin{aligned}
& \text { residue }\left(x_{1}^{p_{1}} x_{2}^{p_{2}} \cdots x_{n}^{p_{n}} F_{1}^{-m_{1}-1} \cdots F_{n}^{-m_{n}-1} \frac{\partial\left(F_{1}, \ldots, F_{n}\right)}{\partial\left(x_{1}, \ldots, x_{n}\right)}\right) \\
& =\operatorname{residue}\left(\sum_{l_{1}, \ldots, l_{n}} d_{l_{1}, \ldots, l_{n}}^{p_{1}, \ldots, p_{n}} F_{1}^{l_{1}-m_{1}-1} \cdots F_{n}^{l_{n}-m_{n}-1} \frac{\partial\left(F_{1}, \ldots, F_{n}\right)}{\partial\left(x_{1}, \ldots, x_{n}\right)}\right) \quad \text { by }(1.3) \\
& =\operatorname{residue}\left(d_{m_{1}, \ldots, m_{n}}^{p_{1}, \ldots, p_{n}} F_{1}^{-1} \cdots F_{n}^{-1} \frac{\partial\left(F_{1}, \ldots, F_{n}\right)}{\partial\left(x_{1}, \ldots, x_{n}\right)}\right) \quad \text { by }(1.2) \text { and Theorem } 1.4 \\
& =d_{m_{1}, \ldots, m_{n}}^{p_{1}, \ldots, p_{n}} \text { by Theorem 1.4. }
\end{aligned}
$$

\section{Power SERIES REVERsion IN TWO VARIABleS}

A 2-colored tree is a (finite) rooted tree in which every node has either color 1 or color 2 and the children of each node are linearly ordered in such a way that nodes of color 1 always precede those of color 2. A $(p, q)$-forest $F$ is an ordered set of $p$ 2 -colored trees with color- 1 roots followed by $q$ 2-colored trees with color- 2 roots. For instance, a (1,0)-forest is simply a 2-colored tree whose root has color 1.

Let $\mathbb{N}$ denote the set of nonnegative integers and let $\boldsymbol{\alpha}=\left(\alpha_{i j}\right)_{j=0,1,2, \ldots}^{i=0,1,2, \ldots}, \boldsymbol{\beta}=$ $\left(\beta_{i j}\right)_{j=0,1,2, \ldots}^{i=0,1,2, \ldots}$ be two matrices over $\mathbb{N}$ with only finitely many nonzero entries. We shall call the ordered pair $(\boldsymbol{\alpha}, \boldsymbol{\beta})$ the inventory of $F$ if $\alpha_{i j}$ equals the number of color- 1 nodes in $F$ with $i$ children of color 1 and $j$ children of color 2, and $\beta_{i j}$ equals the number of color- 2 nodes in $F$ with $i$ children of color 1 and $j$ children of color 2 .

Let

$$
\sigma(\boldsymbol{\alpha})=\sum_{i, j} \alpha_{i j}, \quad \sigma_{1}(\boldsymbol{\alpha})=\sum_{i, j} i \alpha_{i j}
$$

and

$$
\sigma_{2}(\boldsymbol{\alpha})=\sum_{i, j} j \alpha_{i j}
$$

Example 2.0. Consider the 2-colored tree $T$ in Figure 2.1. A round node indicates that it is of color 1 (or female) while a square node indicates that it is of color 2 (or male). Then $\alpha_{i j}$ counts the number of female nodes with $i$ daughters and $j$ sons, and $\beta_{i j}$ counts the number of male nodes with $i$ daughters and $j$ sons. 


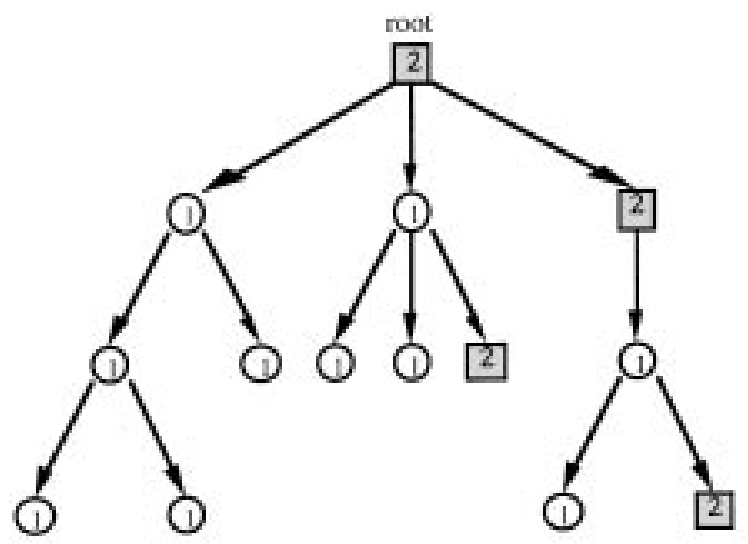

FiguRE 2.1

The inventory of the tree $T$ is

$$
\begin{aligned}
(\boldsymbol{\alpha}, \boldsymbol{\beta}) & =\left(\begin{array}{lllllllll}
\alpha_{00} & \alpha_{01} & \alpha_{02} & \ldots & \vdots & \beta_{00} & \beta_{01} & \beta_{02} & \ldots \\
\alpha_{10} & \alpha_{11} & \alpha_{12} & \ldots & \vdots & \beta_{10} & \beta_{11} & \beta_{12} & \ldots \\
\alpha_{20} & \alpha_{21} & \alpha_{22} & \ldots & \vdots & \beta_{20} & \beta_{21} & \beta_{22} & \ldots \\
\ldots \ldots \ldots \ldots \ldots \ldots \ldots & \vdots & \ldots \ldots \ldots \ldots \ldots \ldots \ldots \\
\ldots \ldots & \ldots \ldots \ldots \ldots & \vdots & \ldots \ldots \ldots \ldots \ldots \ldots
\end{array}\right) \\
& =\left(\begin{array}{lllllll}
6 & 0 & \vdots & 2 & 0 & \\
0 & 1 & \mathbf{0} & \vdots & 1 & 0 & \mathbf{0} \\
2 & 1 & \vdots & 0 & 1 \\
\mathbf{0} & \mathbf{0} & \vdots & \mathbf{0} & \mathbf{0}
\end{array}\right) .
\end{aligned}
$$

Lemma 2.1. If $(\boldsymbol{\alpha}, \boldsymbol{\beta})$ is the inventory of a $(p, q)$-forest $F$, then

$$
\begin{aligned}
& p=\sigma(\boldsymbol{\alpha})-\sigma_{1}(\boldsymbol{\alpha}+\boldsymbol{\beta}), \\
& q=\sigma(\boldsymbol{\beta})-\sigma_{2}(\boldsymbol{\alpha}+\boldsymbol{\beta}) .
\end{aligned}
$$

Proof. Note that $\sum \alpha_{i j}$ is the number of color- 1 nodes in $F$ and $\sum \beta_{i j}$ is the number of color-2 nodes in $F$. Since the number of children of color 1 is equal to $\sum i \alpha_{i j}+\sum i \beta_{i j}$, the first identity follows from the fact that roots are the only nodes that are not children. The second identity can be proved similarly.

Remark. The converse of the above is true if $p q \neq 0$ (see Corollary 3.4). To see that the condition $p q \neq 0$ is necessary, let $p=1, q=0, \alpha_{00}=1, \beta_{01}=1$ with all other $\alpha_{i j}$ 's and $\beta_{i j}$ 's being zero. Then (2.1) is satisfied but $(\boldsymbol{\alpha}, \boldsymbol{\beta})$ is not the inventory of any $(1,0)$-forest.

Given a pair $(\boldsymbol{\alpha}, \boldsymbol{\beta})$ of matrices as before, define $R(\boldsymbol{\alpha}, \boldsymbol{\beta})$ to be the number of $(p, q)$-forests with inventory $(\boldsymbol{\alpha}, \boldsymbol{\beta})$ where $p$ and $q$ are defined by (2.1). For $p \geqslant 0$ and $q \geqslant 0$, we shall denote by $\mathcal{F}_{p, q}$ the set of all $(\boldsymbol{\alpha}, \boldsymbol{\beta})$ satisfying (2.1). Let $X$ and 
$Y$ be the generating functions for the number of $(1,0)$-forests and the number of $(0,1)$-forests respectively, both classified by inventories. Then

$$
\begin{aligned}
X & =\sum_{\mathcal{F}_{1,0}} R(\boldsymbol{\alpha}, \boldsymbol{\beta}) \prod a_{i j}^{\alpha_{i j}} \prod b_{i j}^{\beta_{i j}}, \\
Y & =\sum_{\mathcal{F}_{0,1}} R(\boldsymbol{\alpha}, \boldsymbol{\beta}) \prod a_{i j}^{\alpha_{i j}} \prod b_{i j}^{\beta_{i j}} .
\end{aligned}
$$

The sum in the first equation should be over all inventories of $(1,0)$-forests; however, it can be extended to $\mathcal{F}_{1,0}$ for if $(\boldsymbol{\alpha}, \boldsymbol{\beta})$ is in $\mathcal{F}_{1,0}$ but is not the inventory of any $(1,0)$-forest, then $R(\boldsymbol{\alpha}, \boldsymbol{\beta})=0$. Both $X$ and $Y$ are formal power series in infinitely many variables $a_{i j}, b_{i j}$, where $i, j=0,1,2, \ldots$.

Lemma 2.2. $X^{p} Y^{q}$ is the generating function for the number of $(p, q)$-forests classified by inventories. In fact,

$$
X^{p} Y^{q}=\sum_{\mathcal{F}_{p, q}} R(\boldsymbol{\alpha}, \boldsymbol{\beta}) \prod a_{i j}^{\alpha_{i j}} \prod b_{i j}^{\beta_{i j}} .
$$

Proof. Let $(\boldsymbol{\alpha}, \boldsymbol{\beta})$ be the inventory of a $(p, q)$-forest. Then $R(\boldsymbol{\alpha}, \boldsymbol{\beta})$ is equal to $\sum R\left(\boldsymbol{\alpha}_{1}, \boldsymbol{\beta}_{1}\right) \cdots R\left(\boldsymbol{\alpha}_{p+q}, \boldsymbol{\beta}_{p+q}\right)$ where the sum is over all sequences $\left(\boldsymbol{\alpha}_{1}, \boldsymbol{\beta}_{1}\right), \ldots$, $\left(\boldsymbol{\alpha}_{p+q}, \boldsymbol{\beta}_{p+q}\right)$ such that $\boldsymbol{\alpha}=\sum \boldsymbol{\alpha}_{k}, \boldsymbol{\beta}=\sum \boldsymbol{\beta}_{k}$, and $\left(\boldsymbol{\alpha}_{k}, \boldsymbol{\beta}_{k}\right)$ is the inventory of a $(1,0)$-forest for $k=1, \ldots, p,\left(\boldsymbol{\alpha}_{k}, \boldsymbol{\beta}_{k}\right)$ is the inventory of a $(0,1)$-forest for $k=p+1$, $\ldots, p+q$. Clearly, the sum can be extended to all sequences such that $\boldsymbol{\alpha}=\sum \boldsymbol{\alpha}_{k}$, $\boldsymbol{\beta}=\sum \boldsymbol{\beta}_{k}$, and $\left(\boldsymbol{\alpha}_{k}, \boldsymbol{\beta}_{k}\right)$ is in $\mathcal{F}_{1,0}$ for $k=1, \ldots, p,\left(\boldsymbol{\alpha}_{k}, \boldsymbol{\beta}_{k}\right)$ is in $\mathcal{F}_{0,1}$ for $k=p+1$, $\ldots, p+q$. Thus it follows from (2.2) that $X^{p} Y^{q}$ is the generating function for the number of $(p, q)$-forests.

Lemma 2.3. $X$ and $Y$ of (2.2) satisfy the following functional equations

$$
\begin{aligned}
& X=\sum a_{i j} X^{i} Y^{j}, \\
& Y=\sum b_{i j} X^{i} Y^{j} .
\end{aligned}
$$

Proof. Using Lemma 2.2, we see that $a_{p q} X^{p} Y^{q}$ is the generating function for the number of $(1,0)$-forests the removal of whose roots produces $(p, q)$-forests. Then the first equation follows by partitioning the set of all $(1,0)$-forests into classes by the number $p$ of color-1-children and the number $q$ of color-2-children of the roots. The proof for the second is similar.

Theorem 2.4 (Direct Reversion). Suppose

$$
\begin{aligned}
& F=x-\sum_{i+j \geqslant 2} a_{i j} x^{i} y^{j}, \\
& G=y-\sum_{i+j \geqslant 2} b_{i j} x^{i} y^{j},
\end{aligned}
$$

where the $a_{i j}$ and the $b_{i j}$ are indeterminates. Then, for $p, q \geqslant 0$,

$$
x^{p} y^{q}=\sum_{l, m} e_{l, m}^{p, q} F^{l} G^{m}
$$

where

$$
e_{l, m}^{p, q}=\sum R(\boldsymbol{\alpha}, \boldsymbol{\beta}) \prod_{i+j \geqslant 2} a_{i j}^{\alpha_{i j}} \prod_{i+j \geqslant 2} b_{i j}^{\beta_{i j}}
$$


with the sum indexed by all $(\boldsymbol{\alpha}, \boldsymbol{\beta})$ in $\mathcal{F}_{p, q}$ satisfying the condition that $l=\alpha_{00}$, $m=\beta_{00}$, and $\alpha_{10}=\alpha_{01}=\beta_{10}=\beta_{01}=0$

Proof. Setting $a_{01}=a_{10}=b_{01}=b_{10}=0, X=x, Y=y, a_{00}=F, b_{00}=G$ in (2.2), and using Lemma 2.3, we see that the following satisfies (2.4).

$$
\begin{aligned}
& x=\sum_{l, m}\left(\sum R(\boldsymbol{\alpha}, \boldsymbol{\beta}) \prod_{i+j \geqslant 2} a_{i j}^{\alpha_{i j}} \prod_{i+j \geqslant 2} b_{i j}^{\beta_{i j}}\right) F^{l} G^{m}, \\
& y=\sum_{l, m}\left(\sum R(\boldsymbol{\alpha}, \boldsymbol{\beta}) \prod_{i+j \geqslant 2} a_{i j}^{\alpha_{i j}} \prod_{i+j \geqslant 2} b_{i j}^{\beta_{i j}}\right) F^{l} G^{m},
\end{aligned}
$$

where the first inner sum is indexed by all $(\boldsymbol{\alpha}, \boldsymbol{\beta})$ in $\mathcal{F}_{1,0}$ and the second by $\mathcal{F}_{0,1}$, and in both cases, satisfying the conditions: $l=\alpha_{00}, m=\beta_{00}$ and $\alpha_{10}=\alpha_{01}=$ $\beta_{10}=\beta_{01}=0$. By [2, Chapter III, Section 4.4, Proposition 5, pp. 219-220], a right compositional inverse is also a left inverse in formal power series reversion, so (2.4) also satisfies (2.5). Now the result follows from Lemma 2.2.

Theorem 2.5 (Diagonal Reversion). Suppose

$$
F=\frac{x}{1+\sum_{i+j \geqslant 1} a_{i j} x^{i} y^{j}}, \quad G=\frac{y}{1+\sum_{i+j \geqslant 1} b_{i j} x^{i} y^{j}},
$$

where the $a_{i j}$ and the $b_{i j}$ are indeterminates. Then, for $p, q \geqslant 0$,

$$
x^{p} y^{q}=\sum_{l, m} d_{l, m}^{p, q} F^{l} G^{m}
$$

where

$$
d_{l, m}^{p, q}=\sum R(\boldsymbol{\alpha}, \boldsymbol{\beta}) \prod_{i+j \geqslant 1} a_{i j}^{\alpha_{i j}} \prod_{i+j \geqslant 1} b_{i j}^{\beta_{i j}}
$$

with the sum indexed by all $(\boldsymbol{\alpha}, \boldsymbol{\beta})$ in $\mathcal{F}_{p, q}$ satisfying the conditions $l=\sigma(\boldsymbol{\alpha})$ and $m=\sigma(\boldsymbol{\beta})$.

Proof. Substitute $a_{00} a_{i j}$ for $a_{i j}, b_{00} b_{i j}$ for $b_{i j}$ for all $i+j \geqslant 1$, and then set $a_{00}=F$, $b_{00}=G$ in (2.2). Now proceed as in the proof of Theorem 2.4.

In the following we shall find a formula for $R(\boldsymbol{\alpha}, \boldsymbol{\beta})$ thus determining $e_{l, m}^{p, q}$ and $d_{l, m}^{p, q}$ in Theorems 2.4 and 2.5 respectively. For any matrix $\boldsymbol{\alpha}=\left(\alpha_{i j}\right)_{j=0,1,2, \ldots}^{i=0,1,2, \ldots}$ over $\mathbb{N}$ with a finite number of nonzero entries, we define the multinomial and the "modified" multinomial coefficients by

$$
\begin{aligned}
& M(\boldsymbol{\alpha})=\left(\begin{array}{c}
\sigma(\boldsymbol{\alpha}) \\
\alpha_{00}, \ldots, \alpha_{i j}, \ldots
\end{array}\right)=\frac{\sigma(\boldsymbol{\alpha}) !}{\prod\left(\alpha_{i j} !\right)}, \\
& \widetilde{M}(\boldsymbol{\alpha})= \begin{cases}1, & \text { if all } \alpha_{i j}=0, \\
\frac{1}{\sigma(\boldsymbol{\alpha})} M(\boldsymbol{\alpha}), & \text { otherwise. }\end{cases}
\end{aligned}
$$


Theorem 2.6. Suppose $F$ and $G$ are as in Theorem 2.5. Then

$$
\begin{aligned}
d_{l, m}^{p, q}= & \sum\left|\left(\begin{array}{cc}
M(\boldsymbol{\alpha}) & 0 \\
0 & M(\boldsymbol{\beta})
\end{array}\right)-\left(\begin{array}{cc}
\widetilde{M}(\boldsymbol{\alpha}) & 0 \\
0 & \widetilde{M}(\boldsymbol{\beta})
\end{array}\right)\left(\begin{array}{cc}
\sigma_{1}(\boldsymbol{\alpha}) & \sigma_{2}(\boldsymbol{\alpha}) \\
\sigma_{1}(\boldsymbol{\beta}) & \sigma_{2}(\boldsymbol{\beta})
\end{array}\right)\right| \\
& \times \prod_{i+j \geqslant 1} a_{i j}^{\alpha_{i j}} \prod_{i+j \geqslant 1} b_{i j}^{\beta_{i j}}
\end{aligned}
$$

with the sum indexed by all $(\boldsymbol{\alpha}, \boldsymbol{\beta})$ in $\mathcal{F}_{p, q}$ satisfying the conditions $l=\sigma(\boldsymbol{\alpha})$ and $m=\sigma(\boldsymbol{\beta})$.

Proof. It suffices to prove

$$
\begin{aligned}
x^{p} y^{q}\left|\begin{array}{rr}
F^{-l-1} \frac{\partial F}{\partial x} & F^{-l-1} \frac{\partial F}{\partial y} \\
G^{-m-1} \frac{\partial G}{\partial x} & G^{-m-1} \frac{\partial G}{\partial y}
\end{array}\right| \\
=\sum_{\substack{\boldsymbol{\alpha}, \boldsymbol{\beta} \\
\sigma(\boldsymbol{\alpha})=l \\
\sigma(\boldsymbol{\beta})=m}} x^{\sigma_{1}(\boldsymbol{\alpha}+\boldsymbol{\beta})-\sigma(\boldsymbol{\alpha})+p-1} y^{\sigma_{2}(\boldsymbol{\alpha}+\boldsymbol{\beta})-\sigma(\boldsymbol{\beta})+q-1} \\
\times\left|\begin{array}{rr}
M(\boldsymbol{\alpha})-\widetilde{M}(\boldsymbol{\alpha}) \sigma_{1}(\boldsymbol{\alpha}) & 0-\widetilde{M}(\boldsymbol{\alpha}) \sigma_{2}(\boldsymbol{\alpha}) \\
0-\widetilde{M}(\boldsymbol{\beta}) \sigma_{1}(\boldsymbol{\beta}) & M(\boldsymbol{\beta})-\widetilde{M}(\boldsymbol{\beta}) \sigma_{2}(\boldsymbol{\beta})
\end{array}\right| \mathbf{A}^{\boldsymbol{\alpha}} \mathbf{B}^{\boldsymbol{\beta}},
\end{aligned}
$$

since the conclusion of Theorem 2.6 follows from taking the residue of (2.6) and using Corollary 1.5.

Let $H=1+\sum_{i+j \geqslant 1} a_{i j} x^{i} y^{j}$. Then $F=\frac{x}{H}$ and so,

$$
\frac{\partial F}{\partial x}=\frac{1}{H}-\frac{x}{H^{2}} \frac{\partial H}{\partial x}=\frac{F}{x}-\frac{F^{2}}{x} \frac{\partial H}{\partial x} .
$$

Hence

$$
F^{-l-1} \frac{\partial F}{\partial x}=\frac{F^{-l}}{x}-\frac{F^{-l+1}}{x} \frac{\partial H}{\partial x} .
$$

By binomial and multinomial expansions, we have

$$
\begin{aligned}
\left(1+\sum_{i+j \geqslant 1} a_{i j} x^{i} y^{j}\right)^{l} & =\sum_{k=0}^{l}\left(\begin{array}{l}
l \\
k
\end{array}\right)\left(\sum_{i+j \geqslant 1} a_{i j} x^{i} y^{j}\right)^{k} \\
& =\sum_{k=0}^{l}\left(\begin{array}{l}
l \\
k
\end{array}\right) \sum_{\substack{\boldsymbol{\alpha}^{\prime} \\
\sigma\left(\boldsymbol{\alpha}^{\prime}\right)=k}}\left(\begin{array}{c}
k \\
\boldsymbol{\alpha}^{\prime}
\end{array}\right) \prod_{i+j \geqslant 1}\left(a_{i j} x^{i} y^{j}\right)^{\alpha_{i j}^{\prime}} \\
& =\sum_{\substack{\boldsymbol{\alpha}^{\prime} \\
\sigma\left(\boldsymbol{\alpha}^{\prime}\right) \leqslant l}}\left(l-\sigma\left(\boldsymbol{\alpha}^{\prime}\right), \alpha_{01}^{\prime}, \alpha_{10}^{\prime}, \ldots, \alpha_{i j}^{\prime}, \ldots\right) \prod_{i+j \geqslant 1}\left(a_{i j} x^{i} y^{j}\right)^{\alpha_{i j}^{\prime}}
\end{aligned}
$$

where $\boldsymbol{\alpha}^{\prime}=\left(\alpha_{i j}^{\prime}\right)_{i+j \geqslant 1}$. Extend $\boldsymbol{\alpha}^{\prime}$ to $\boldsymbol{\alpha}=\left(\alpha_{i j}\right)_{i+j \geqslant 0}$ by letting $\alpha_{00}=l-\sigma\left(\boldsymbol{\alpha}^{\prime}\right)$ and $\alpha_{i j}=\alpha_{i j}^{\prime}$ for $i+j \geqslant 1$. Then $\sigma_{1}(\boldsymbol{\alpha})=\sigma_{1}\left(\boldsymbol{\alpha}^{\prime}\right), \sigma_{2}(\boldsymbol{\alpha})=\sigma_{2}\left(\boldsymbol{\alpha}^{\prime}\right)$ and $\sigma(\boldsymbol{\alpha})=l$. 
Thus the last expression equals

$$
\sum_{\substack{\boldsymbol{\alpha} \\
\sigma(\boldsymbol{\alpha})=l}}\left(\begin{array}{c}
l \\
\alpha_{00}, \alpha_{01}, \alpha_{10}, \ldots, \alpha_{i j}, \ldots
\end{array}\right) \prod_{i+j \geqslant 1}\left(a_{i j} x^{i} y^{j}\right)^{\alpha_{i j}}
$$

and so

$$
F^{-l}=\sum_{\substack{\boldsymbol{\alpha} \\ \sigma(\boldsymbol{\alpha})=l}} M(\boldsymbol{\alpha}) x^{\sigma_{1}(\boldsymbol{\alpha})-\sigma(\boldsymbol{\alpha})} y^{\sigma_{2}(\boldsymbol{\alpha})} \mathbf{A}^{\boldsymbol{\alpha}}
$$

where $\mathbf{A}^{\alpha}=\prod_{i+j \geqslant 1} a_{i j}^{\alpha_{i j}}$.

Now, let $\varepsilon(i, j)$ denote the matrix whose only nonzero entry is 1 and which occurs at the $(i, j)$-position. Then

$$
\frac{\partial H}{\partial x}=\sum_{i+j \geqslant 1} i x^{i-1} y^{j} \mathbf{A}^{\boldsymbol{\varepsilon}(i, j)}
$$

Using (2.8), we see that

$$
\frac{F^{-l+1}}{x} \frac{\partial H}{\partial x}=\sum_{i+j \geqslant 1} \sum_{\substack{\boldsymbol{\alpha}^{\prime} \\ \sigma\left(\boldsymbol{\alpha}^{\prime}\right)=l-1}} i M\left(\boldsymbol{\alpha}^{\prime}\right) x^{\sigma_{1}\left(\boldsymbol{\alpha}^{\prime}\right)-\sigma\left(\boldsymbol{\alpha}^{\prime}\right)-1+i-1} y^{\sigma_{2}\left(\boldsymbol{\alpha}^{\prime}\right)+j} \mathbf{A}^{\boldsymbol{\alpha}^{\prime}} \mathbf{A}^{\boldsymbol{\varepsilon}(i, j)}
$$

For each fixed pair $(i, j)$, let $\boldsymbol{\alpha}=\boldsymbol{\alpha}^{\prime}+\varepsilon(i, j)$. Then $\alpha_{i j} \neq 0, \sigma_{1}\left(\boldsymbol{\alpha}^{\prime}\right)+i=\sigma_{1}(\boldsymbol{\alpha})$, $\sigma_{2}\left(\boldsymbol{\alpha}^{\prime}\right)+j=\sigma_{2}(\boldsymbol{\alpha}), \sigma\left(\boldsymbol{\alpha}^{\prime}\right)+1=\sigma(\boldsymbol{\alpha})$, and $M\left(\boldsymbol{\alpha}^{\prime}\right)=\alpha_{i j} \widetilde{M}(\boldsymbol{\alpha})$. Hence

$$
\begin{aligned}
\frac{F^{-l+1}}{x} \frac{\partial H}{\partial x} & =\sum_{i+j \geqslant 1} \sum_{\substack{\boldsymbol{\alpha}(\boldsymbol{\alpha})=l \\
\alpha_{i j} \neq 0}} i \alpha_{i j} \widetilde{M}(\boldsymbol{\alpha}) x^{\sigma_{1}(\boldsymbol{\alpha})-\sigma(\boldsymbol{\alpha})-1} y^{\sigma_{2}(\boldsymbol{\alpha})} \mathbf{A}^{\boldsymbol{\alpha}} \\
& =\sum_{\substack{\boldsymbol{\alpha} \\
\sigma(\boldsymbol{\alpha})=l}} \widetilde{M}(\boldsymbol{\alpha}) \sigma_{1}(\boldsymbol{\alpha}) x^{\sigma_{1}(\boldsymbol{\alpha})-\sigma(\boldsymbol{\alpha})-1} y^{\sigma_{2}(\boldsymbol{\alpha})} \mathbf{A}^{\boldsymbol{\alpha}} .
\end{aligned}
$$

The last expression is obtained by noting the condition $\alpha_{i j} \neq 0$ for the inner sum is redundant as $\alpha_{i j}$ is a factor of the summand, and using the definition of $\sigma_{1}(\boldsymbol{\alpha})$ after the summation signs are interchanged. Hence it follows from (2.7) and (2.8) that

$$
F^{-l-1} \frac{\partial F}{\partial x}=\sum_{\substack{\boldsymbol{\alpha} \\ \sigma(\boldsymbol{\alpha})=l}}\left[M(\boldsymbol{\alpha})-\widetilde{M}(\boldsymbol{\alpha}) \sigma_{1}(\alpha)\right] x^{\sigma_{1}(\boldsymbol{\alpha})-\sigma(\boldsymbol{\alpha})-1} y^{\sigma_{2}(\boldsymbol{\alpha})} \mathbf{A}^{\boldsymbol{\alpha}}
$$

Since

$$
\frac{\partial F}{\partial y}=0-\frac{F^{2}}{x} \frac{\partial H}{\partial y}
$$

we may proceed as before to obtain

$$
F^{-l-1} \frac{\partial F}{\partial y}=\sum_{\substack{\boldsymbol{\alpha} \\ \sigma(\boldsymbol{\alpha})=l}}\left[0-\widetilde{M}(\boldsymbol{\alpha}) \sigma_{2}(\alpha)\right] x^{\sigma_{1}(\boldsymbol{\alpha})-\sigma(\boldsymbol{\alpha})} y^{\sigma_{2}(\boldsymbol{\alpha})-1} \mathbf{A}^{\boldsymbol{\alpha}}
$$


Likewise, we have

$$
\begin{aligned}
G^{-m} & =\sum_{\substack{\boldsymbol{\beta} \\
\sigma(\boldsymbol{\beta})=m}} M(\boldsymbol{\beta}) x^{\sigma_{1}(\boldsymbol{\beta})} y^{\sigma_{2}(\boldsymbol{\beta})-\sigma(\boldsymbol{\beta})} \mathbf{B}^{\boldsymbol{\beta}}, \\
G^{-m-1} \frac{\partial G}{\partial x} & =\sum_{\substack{\boldsymbol{\beta} \\
\sigma(\boldsymbol{\beta})=m}}\left[0-\widetilde{M}(\boldsymbol{\beta}) \sigma_{1}(\beta)\right] x^{\sigma_{1}(\boldsymbol{\beta})-1} y^{\sigma_{2}(\boldsymbol{\beta})-\sigma(\boldsymbol{\beta})} \mathbf{B}^{\boldsymbol{\beta}}, \\
G^{-m-1} \frac{\partial G}{\partial y} & =\sum_{\substack{\boldsymbol{\beta} \\
\sigma(\boldsymbol{\beta})=m}}\left[M(\boldsymbol{\beta})-\widetilde{M}(\boldsymbol{\beta}) \sigma_{2}(\beta)\right] x^{\sigma_{1}(\boldsymbol{\beta})} y^{\sigma_{2}(\boldsymbol{\beta})-\sigma(\boldsymbol{\beta})-1} \mathbf{B}^{\boldsymbol{\beta}} .
\end{aligned}
$$

Now (2.6) follows from (2.9), (2.10), (2.11) and (2.12).

Corollary 2.7. For any $(\boldsymbol{\alpha}, \boldsymbol{\beta}) \in \mathcal{F}_{p, q}$,

$$
R(\boldsymbol{\alpha}, \boldsymbol{\beta})=\left|\left(\begin{array}{cc}
M(\boldsymbol{\alpha}) & 0 \\
0 & M(\boldsymbol{\beta})
\end{array}\right)-\left(\begin{array}{cc}
\widetilde{M}(\boldsymbol{\alpha}) & 0 \\
0 & \widetilde{M}(\boldsymbol{\beta})
\end{array}\right)\left(\begin{array}{cc}
\sigma_{1}(\boldsymbol{\alpha}) & \sigma_{2}(\boldsymbol{\alpha}) \\
\sigma_{1}(\boldsymbol{\beta}) & \sigma_{2}(\boldsymbol{\beta})
\end{array}\right)\right| .
$$

Proof. Compare the results of Theorems 2.5 and 2.6.

Remark. Using Corollary 2.7 we can now determine the $e_{l, m}^{p, q}$ in Theorem 2.4.

\section{Generalizations}

Using essentially the same proofs, all results in Section 2 can be generalized to an arbitrary number of colors. In what follows, we shall indicate how this can be done.

An $n$-colored tree is a (finite) rooted tree in which every node has one of the $n$ colors and the children of each node are linearly ordered in such a way that children of color $i$ always precede those of color $j$ if $i<j$. A $\left(p_{1}, \ldots, p_{n}\right)$-forest is an ordered set of $n$-colored trees with $p_{i}$ trees of root-color $i$ for $i=1, \ldots, n$ such that trees with root-color $i$ always precede those of root-color $j$ if $i<j$.

For $i=1, \ldots, n$, let $\boldsymbol{\alpha}^{(i)}=\left(\alpha_{k_{1}, \ldots, k_{n}}^{(i)}\right)_{k_{1}, \ldots, k_{n} \in \mathbb{N}}$ be an $n$-dimensional array over $\mathbb{N}$ with finitely many nonzero entries. If, for each $i$ and for each $n$-tuple $\left(k_{1}, \ldots, k_{n}\right)$, $\alpha_{k_{1}, \ldots, k_{n}}^{(i)}$ equals the number of color $i$ nodes in a $\left(p_{1}, \ldots, p_{n}\right)$-forest $F$ with $k_{j}$ children of color $j$ for each $j=1, \ldots, n$, then $\widehat{\boldsymbol{\alpha}}=\left(\boldsymbol{\alpha}^{(1)}, \ldots, \boldsymbol{\alpha}^{(n)}\right)$ is said to be the inventory of $F$.

For any $n$-dimensional array $\boldsymbol{\alpha}=\left(\alpha_{k_{1}, \ldots, k_{n}}\right)_{k_{1}, \ldots, k_{n} \in \mathbb{N}}$ over $\mathbb{N}$ with finitely many nonzero entries, define $\sigma(\boldsymbol{\alpha})=\sum \alpha_{k_{1}, \ldots, k_{n}}$ and $\sigma_{i}(\boldsymbol{\alpha})=\sum k_{i} \alpha_{k_{1}, \ldots, k_{n}}$, for each $i$.

As in the case of Lemma 2.1, if $\widehat{\boldsymbol{\alpha}}=\left(\boldsymbol{\alpha}^{(1)}, \ldots, \boldsymbol{\alpha}^{(n)}\right)$ is the inventory of a $\left(p_{1}, \ldots, p_{n}\right)$-forest, then, for each $i$,

$$
p_{i}=\sigma\left(\boldsymbol{\alpha}^{(i)}\right)-\sigma_{i}\left(\boldsymbol{\alpha}^{(1)}+\cdots+\boldsymbol{\alpha}^{(n)}\right) .
$$

Given an $n$-tuple $\widehat{\boldsymbol{\alpha}}=\left(\boldsymbol{\alpha}^{(1)}, \ldots, \boldsymbol{\alpha}^{(n)}\right)$ of $n$-dimensional arrays, let $R(\widehat{\boldsymbol{\alpha}})$ be the number of $\left(p_{1}, \ldots, p_{n}\right)$-forests with inventory $\widehat{\boldsymbol{\alpha}}$, where each $p_{i}$ is defined by (3.1). For $p_{1}, \ldots, p_{n} \in \mathbb{N}$, we shall also denote by $\mathcal{F}_{p_{1}, \ldots, p_{n}}$ the set of all $\widehat{\boldsymbol{\alpha}}=\left(\boldsymbol{\alpha}^{(1)}, \ldots, \boldsymbol{\alpha}^{(n)}\right)$ satisfying (3.1). For each $i$, let $X_{i}$ be the generating function for the number of 
$\mathbf{e}_{i}$-forests classified by inventories. Here, $\mathbf{e}_{i}=(0, \ldots, 0,1,0, \ldots, 0)$, the 1 being in the $i^{\text {th }}$ position. Then

$$
X_{i}=\sum_{\mathcal{F}_{\mathbf{e}_{i}}} R(\widehat{\boldsymbol{\alpha}}) \prod_{j=1}^{n} \prod_{k_{1}, k_{2}, \ldots, k_{n}} a_{k_{1}, k_{2}, \ldots, k_{n}}^{(j)} \alpha_{k_{1}, k_{2}, \ldots, k_{n}}^{(j)} .
$$

One can show, as in Lemma 2.2, that $X_{1}^{p_{1}} X_{2}^{p_{2}} \cdots X_{n}^{p_{n}}$ is the generating function for the number of $\left(p_{1}, \ldots, p_{n}\right)$-forests classified by inventories. Thus, as in Lemma $2.3, X_{i}$ satisfies the functional equation

$$
X_{i}=\sum a_{k_{1}, \ldots, k_{n}}^{(i)} X_{1}^{k_{1}} X_{2}^{k_{2}} \cdots X_{n}^{k_{n}},
$$

for $i=1, \ldots, n$.

We can now state our main results for the general case of arbitrary $n$.

Theorem 3.1 (Direct Reversion). For each $i=1, \ldots, n$, let

$$
F_{i}=x_{i}-\sum_{k_{1}+\cdots+k_{n} \geqslant 2} a_{k_{1}, \ldots, k_{n}}^{(i)} x_{1}^{k_{1}} x_{2}^{k_{2}} \cdots x_{n}^{k_{n}},
$$

where the $a_{k_{1}, \ldots, k_{n}}^{(i)}$ are indeterminates. Then, for $p_{1}, \ldots, p_{n} \in \mathbb{N}$,

$$
x_{1}^{p_{1}} x_{2}^{p_{2}} \cdots x_{n}^{p_{n}}=\sum_{r_{1}, \ldots, r_{n}} e_{r_{1}, \ldots, r_{n}}^{p_{1}, \ldots, p_{n}} F_{1}^{r_{1}} F_{2}^{r_{2}} \cdots F_{n}^{r_{n}},
$$

where

$$
e_{r_{1}, \ldots, r_{n}}^{p_{1}, \ldots, p_{n}}=\sum R(\widehat{\boldsymbol{\alpha}}) \prod_{j=1}^{n} \prod_{k_{1}+\cdots+k_{n} \geqslant 2} a_{k_{1}, \ldots, k_{n}}^{(j)}{ }^{\alpha_{k_{1}, \ldots, k_{n}}^{(j)}},
$$

with the sum indexed by all $\widehat{\boldsymbol{\alpha}}=\left(\boldsymbol{\alpha}^{(1)}, \ldots, \boldsymbol{\alpha}^{(n)}\right)$ in $\mathcal{F}_{p_{1}, \ldots, p_{n}}$ satisfying the conditions that $r_{i}=\alpha_{0, \ldots, 0}^{(i)}$ and $\alpha_{\mathbf{e}_{j}}^{(i)}=0$ for all $i$ and $j$.

Theorem 3.2 (Diagonal Reversion). For each $i=1, \ldots, n$, let

$$
F_{i}=\frac{x_{i}}{1+\sum_{k_{1}+\cdots+k_{n} \geqslant 1} a_{k_{1}, \ldots, k_{n}}^{(i)} x_{1}^{k_{1}} x_{2}^{k_{2}} \cdots x_{n}^{k_{n}}} .
$$

Then, for $p_{1}, \ldots, p_{n} \in \mathbb{N}$,

$$
x_{1}^{p_{1}} x_{2}^{p_{2}} \cdots x_{n}^{p_{n}}=\sum_{r_{1}, \ldots, r_{n}} d_{r_{1}, \ldots, r_{n}}^{p_{1}, \ldots, p_{n}} F_{1}^{r_{1}} F_{2}^{r_{2}} \cdots F_{n}^{r_{n}},
$$

where

$$
d_{r_{1}, \ldots, r_{n}}^{p_{1}, \ldots, p_{n}}=\sum R(\widehat{\boldsymbol{\alpha}}) \prod_{j=1}^{n} \prod_{k_{1}+\cdots+k_{n} \geqslant 1} a_{k_{1}, \ldots, k_{n}}^{(j)}{ }^{\alpha_{k_{1}, \ldots, k_{n}}^{(j)}},
$$

with the sum indexed by all $\widehat{\boldsymbol{\alpha}}=\left(\boldsymbol{\alpha}^{(1)}, \ldots, \boldsymbol{\alpha}^{(n)}\right)$ in $\mathcal{F}_{p_{1}, \ldots, p_{n}}$ satisfying the conditions that $r_{i}=\sigma\left(\boldsymbol{\alpha}^{(i)}\right)$, for all $i$. 
Theorem 3.3 (Extended Raney Coefficient). For any $\widehat{\boldsymbol{\alpha}}=\left(\boldsymbol{\alpha}^{(1)}, \ldots, \boldsymbol{\alpha}^{(n)}\right) \in$ $\mathcal{F}_{p_{1}, \ldots, p_{n}}$,

$$
\begin{aligned}
R(\widehat{\boldsymbol{\alpha}}) & =\mid\left(\begin{array}{ccc}
M\left(\boldsymbol{\alpha}^{(1)}\right) & & \mathbf{0} \\
& \mathbf{O}^{\ddots} & \\
& & M\left(\boldsymbol{\alpha}^{(n)}\right)
\end{array}\right) \\
& -\left(\begin{array}{cccc}
\widetilde{M}\left(\boldsymbol{\alpha}^{(1)}\right) & & \mathbf{0} \\
\mathbf{0} & \ddots & \\
& & \widetilde{M}\left(\boldsymbol{\alpha}^{(n)}\right)
\end{array}\right)\left(\begin{array}{ccc}
\sigma_{1}\left(\boldsymbol{\alpha}^{(1)}\right) & \ldots & \sigma_{n}\left(\boldsymbol{\alpha}^{(1)}\right) \\
\vdots & \ddots & \vdots \\
\sigma_{1}\left(\boldsymbol{\alpha}^{(n)}\right) & \ldots & \sigma_{n}\left(\boldsymbol{\alpha}^{(n)}\right)
\end{array}\right) \mid
\end{aligned}
$$

Corollary 3.4. Let $\widehat{\boldsymbol{\alpha}}=\left(\boldsymbol{\alpha}^{(1)}, \ldots, \boldsymbol{\alpha}^{(n)}\right) \in \mathcal{F}_{p_{1}, \ldots, p_{n}}$. If all $p_{i}>0$, then $R(\widehat{\boldsymbol{\alpha}})>0$.

Proof. If each $p_{i}>0$, then, by (3.1), none of $\boldsymbol{\alpha}^{(1)}, \ldots, \boldsymbol{\alpha}^{(n)}$ consists entirely of 0's; hence, $M\left(\boldsymbol{\alpha}^{(i)}\right)=\sigma\left(\boldsymbol{\alpha}^{(i)}\right) \widetilde{M}\left(\boldsymbol{\alpha}^{(i)}\right)$, for all $i$. Thus, by Theorem 3.3,

$$
\begin{aligned}
R(\widehat{\boldsymbol{\alpha}})= & \prod_{i=1, \ldots, n} \widetilde{M}\left(\boldsymbol{\alpha}^{(i)}\right) \\
& \times\left|\begin{array}{cccc}
\sigma\left(\boldsymbol{\alpha}^{(1)}\right)-\sigma_{1}\left(\boldsymbol{\alpha}^{(1)}\right) & -\sigma_{2}\left(\boldsymbol{\alpha}^{(1)}\right) & \ldots & \\
-\sigma_{1}\left(\boldsymbol{\alpha}^{(2)}\right) & \sigma\left(\boldsymbol{\alpha}^{(2)}\right)-\sigma_{2}\left(\boldsymbol{\alpha}^{(2)}\right) & \ldots & -\sigma_{n}\left(\boldsymbol{\alpha}^{(1)}\right) \\
\vdots & \vdots & \ddots & -\sigma_{n}\left(\boldsymbol{\alpha}^{(2)}\right) \\
-\sigma_{1}\left(\boldsymbol{\alpha}^{(n)}\right) & -\sigma_{2}\left(\boldsymbol{\alpha}^{(n)}\right) & \ldots & \sigma\left(\boldsymbol{\alpha}^{(n)}\right)-\sigma_{n}\left(\boldsymbol{\alpha}^{(n)}\right)
\end{array}\right| .
\end{aligned}
$$

The result now follows from Lemma 3.5 below.

Lemma 3.5. Suppose $B$ is a square matrix of order $n$ with real entries $b_{i j}$ such that $b_{i i} \geqslant 0$, for all $i$, and $b_{i j} \leqslant 0$, for $i \neq j$. If all the column-sums of $B$ are nonnegative, then so is the determinant of $B$. If, furthermore, all the column-sums of $B$ are positive, then so is the determinant of $B$.

Proof. By hypothesis, the $j^{\text {th }}$ column-sum $p_{j}=\sum_{i=1}^{n} b_{i j} \geqslant 0$, for all $j$. Thus we can write $B$ as a sum of two matrices with one of them diagonal, namely,

$$
\begin{aligned}
& B=P+B^{\prime} \\
& =\left(\begin{array}{cccccc}
p_{1} & & & \\
& & & \mathbf{0} \\
& p_{2} & & \\
& \mathbf{O} & \ddots & \\
& & & p_{n}
\end{array}\right)+\left(\begin{array}{cccc}
-\sum_{k \neq 1} b_{k 1} & b_{12} & \ldots & b_{1 n} \\
b_{21} & -\sum_{k \neq 2} b_{k 2} & \ldots & b_{2 n} \\
\vdots & \vdots & \ddots & \vdots \\
b_{n 1} & b_{n 2} & \ldots & -\sum_{k \neq n} b_{k n}
\end{array}\right) .
\end{aligned}
$$

Then

$$
\begin{aligned}
\operatorname{det} B & =p_{1} p_{2} \cdots p_{n}+\left(\sum_{1 \leqslant i_{1}<i_{2}<\cdots<i_{l} \leqslant n-1} c_{i_{1}, \ldots, i_{l}} p_{i_{1}} p_{i_{2}} \cdots p_{i_{l}}\right)+\operatorname{det} B^{\prime} \\
& =p_{1} p_{2} \cdots p_{n}+\sum_{1 \leqslant i_{1}<i_{2}<\cdots<i_{l} \leqslant n-1} c_{i_{1}, \ldots, i_{l}} p_{i_{1}} p_{i_{2}} \cdots p_{i_{l}},
\end{aligned}
$$


a polynomial in $p_{1}, \ldots, p_{n}$, where $c_{i_{1}, \ldots, i_{l}}$ is the principal minor of $B^{\prime}$ obtained by deleting the $i_{j}^{\text {th }}$ row and column of $B^{\prime}$, for $j=1,2, \ldots, l$. We only need to show that $c_{i_{1}, \ldots, i_{l}} \geqslant 0$, for all $l=1,2, \ldots, n-1$. However, this follows from the induction hypothesis, since $B^{\prime}$ as well as all its principal submatrices satisfy the hypotheses of Lemma 3.5.

\section{REFERENCES}

1. H. Bass, E. Connell and D. Wright, The Jacobian conjecture: Reduction of degree and formal expansion of the inverse, Bulletin (New Series) of the American Mathematical Society 7 (1982), 287-330, MR 83k:14028. Zbl.539.13012.

2. N. Bourbaki, Commutative Algebra, Addison-Wesley, Reading, Massachusetts, 1972, MR 50:12997. Zbl.279.13001.

3. I. J. Good, Generalizations to several variables of Lagrange's expansion, with applications to stochastic processes, Proceedings of the Cambridge Philosophical Society 56 (1960), 367-380, MR 23:A352. Zbl.135.18802.

4. M. Haiman and W. Schmitt, Incidence algebra antipodes and Lagrange inversion in one and several variables, Journal of Combinatorial Theory, Series A 50 (1989), 172-185, MR 90f:05005. Zbl.747.05007.

5. C. G. J. Jacobi, De resolutione aequationum per series infinitas, Journal für die reine und angewandte Mathematik 6 (1830), 257-286.

6. D. E. Knuth, The Art of Computer Programming, Vol. 1: Fundamental Algorithms, second edition, Addison-Wesley, Reading, Massachusetts, 1973, MR 44:3530; MR 51:14624; MR $\mathbf{5 7 : 1 8 1 6 9 .}$

7. G. N. Raney, Functional composition patterns and power series reversion, Transactions of the American Mathematical Society 94 (1960), 441-451, MR 22:5584. Zbl.131,14.

8. J. Towber, A combinatorial conjecture which implies the Jacobian conjecture (to appear).

9. S. S.-S. Wang, A Jacobian criterion for separability, Journal of Algebra 65 (1980), 453-494, MR 83e:14010. Zbl.471.13005.

10. D. Wright, The tree formulas for reversion of power series, Journal of Pure and Applied Algebra 57 (1989), 191-211, MR 90d:13008. Zbl.672.13010.

Department of Mathematical Sciences, Oakland University, Rochester, Michigan 48309-4401

E-mail address: cheng@vela.acs.oakland.edu

Department of Mathematical Sciences, Oakland University, Rochester, Michigan 48309-4401

E-mail address: mckay@vela.acs.oakland.edu

Department of Mathematics, DePaul University, Chicago, Illinois 60614-3504

E-mail address: matjt@depaul.edu

Department of Mathematical Sciences, Oakland University, Rochester, Michigan 48309-4401

E-mail address: swang@vela.acs.oakland.edu

Department of Mathematics, Washington University, St. Louis, Missouri 63130-4899

E-mail address: wright@einstein.wustl.edu 